\title{
Stability analysis of time-varying discrete interval systems
}

\author{
Karel Sladký \\ Academy of Sciences of the Czech Republic, \\ Institute of Information Theory and Automation \\ P. O. Box 18, CZ-18208 Prague 8, Czech Republic. Tel: 42-2-66414906. \\ Fax: 42-2-66414903. e-mail: sladky@utia.cas.cz
}

\begin{abstract}
In this article necessary and sufficient conditions for the stability of time-varying discrete interval systems are studied and the corresponding stability margins are obtained. Our analysis heavily employs the Perron-Frobenius theorem for nonnegative matrices and its extensions to a class of interval matrices. We show that by combining the approaches suggested in Bauer et al. (1993) and Han and Lee (1994) the existing tests on stability and stability margins both of time-varying and time-invariant discrete interval systems can be considerably improved. The obtained results are tested on several numerical examples.
\end{abstract}

\section{Keywords}

stability of linear systems, interval matrices, nonhomogeneous matrix products

\section{INTRODUCTION}

An interval matrix is a real matrix in which all the elements are known only to the extent that each belongs to specified closed interval. In particular, an $r \times r$ interval matrix $A_{I}$ is in fact a set of real matrices

$$
A_{I}=\left\{A=\left[a_{i j}\right]: a_{i j} \in\left[b_{i j}, c_{i j}\right], \quad i, j=1, \ldots, r\right\}
$$

where $b_{i j} \leq c_{i j}$ are given real numbers. Let $B=\left[b_{i j}\right], C=\left[c_{i j}\right]$, and hence $A_{I}=\left[\begin{array}{ll}B & C\end{array}\right]$.

The time-varying discrete system is given for $k=0,1, \ldots$, by

$x(k+1)=A(k) x(k), \quad x(0)=x_{0}$

where $x(k)=\left[x_{i}(k)\right]$ is an $r$-dimensional state vector and $A(k) \in A_{I}$.

The system (1) is (globally) asymptotically stable if for every $x(0)=x_{0}$ and every matrix sequence $\left\{A(k), k=0,1, \ldots: A(k) \in A_{I}\right\} \lim _{k \rightarrow \infty} x(k)=0$. The time-varying system (1) is said to be stable with stability margin $h$, where $0 \leq h<1$ (or to have the degree of 
stability $h$ ), if there exists a number $c \geq 0$ (depending on $x_{0}$ ) such that for an arbitrary matrix sequence $A(k) \in A_{I}$ it holds that $\left|x_{i}(k)\right| \leq c(1-h)^{k}$ for $i=1, \ldots, r$. Observe that from our definition of the stability margin we immediately conclude that the timeinvariant discrete system (1) (i. e. if in (1) $A(k) \equiv A \in A_{I}, \forall k=0,1, \ldots$ ) is stable with stability margin $h$ if and only if the modulus of every eigenvalue of any $A \in A_{I}$ is less than $1-h$. A matrix $A \in A_{I}$ is called extreme (vertex) if for every $i, j=1,2, \ldots, r$ either $a_{i j}=b_{i j}$ or $a_{i j}=c_{i j}$, and is (asymptotically) stable iff the system $x(k+1)=A x(k), \forall k$ is (asymptotically) stable. Considering some $A \in A_{I}$, the symbol $|A|$ is reserved for the corresponding modulus matrix, i. e. $|A|=\left\{\left|a_{i j}\right|\right]$, where $\left|a_{i j}\right|$ is the absolute value of $a_{i j}$. In the sequel, we denote by $\bar{A}_{I}$ the (finite) set of all extreme matrices. Obviously, $\bar{A}_{I} \subset A_{I}$ and $\bar{A}_{I}$ possesses not more than $2^{r^{2}}$ different matrices. An extreme matrix $\hat{A} \in \bar{A}_{I}$ is called dominating if $|\hat{A}| \geq|A|$ for any $A \in \bar{A}_{I}$ (obviously then also $|\hat{A}| \geq|A|$ for any $A \in A_{I}$ ).

The stability analysis of dynamic interval systems is very important in the robust controller design. In recent years, stability of dynamic interval systems has been studied by many authors and some sufficients conditions for the stability have been obtained mostly for time-invariant interval systems. In particular, Jiang (1988) has attempted to show that the time-invariant discrete system (1) is asymptotically stable, if and only if all extreme matrices are asymptotically stable. This result was shown to be incorrect (cf. e.g. Kolla and Farison (1988) and the Correspondence in International Journal of Control (1989)). However, it was shown in Mori and Kokame (1987) and in Juang et al. (1989a) that the time-invariant system (1) is asymptotically stable if the norm of the extreme matrices is restricted to be less than unity.

In the present contribution we focus our attention on the stability of time-varying interval systems. This topic was recently studied in Bauer et al. (1993) and in Han and Lee (1994). In Bauer et al. (1993) a necessary and sufficient condition for the stability of time-varying discrete interval matrices was presented. The condition is based on checking and evaluating the products of extreme matrices using standard matrix norms. Even the authors presented an algorithmic procedure for generating and checking these products, there are severe computational limits of an actual implementation of the algorithm since the computational complexity increases exponentially with the size of the considered interval matrix (recall that the number of extreme matrices from $\bar{A}_{I}$ equals $2^{r^{2}}$ ). On the other hand in Han and Lee (1994) a simple sufficient condition for the stability of timevarying discrete interval systems was presented. We show that the sufficient condition on stability of time-varying discrete interval systems presented in Han and Lee (1994) can be easily obtained from the Perron-Frobenius theorem on maximal eigenvalue of a nonnegative matrix. The simple test on stability suggested in Han and Lee (1994) can be further amplified by combining this test with the approach presented in Bauer et al. (1993) and by evaluating the resulting matrices by weighted matrix norms. The obtained results are also useful for improving existing stability results on time-invariant systems.

\section{PRELIMINARIES}

Recall that, according to the well-known Perron-Frobenius theorem (cf. e.g. Gantmacher (1966), Berman and Plemmons (1979)), the spectral radius, denoted $\rho(\cdot)$, of a nonnegative matrix is equal to its largest positive eigenvalue (called the Perron eigenvalue) 
and the corresponding left, right eigenvector (denoted $v(\cdot), u(\cdot)$ and called the left, right Perron eigenvector respectively) can be selected nonnegative. In case that the matrix is irreducible, the Perron eigenvalue is simple, the Perron eigenvector is unique up to a multiplicative constant and can be selected positive.

In particular, for the dominating matrix $\hat{A}$ it holds (remember that symbols $\geq,>$ in a matrix relation are considered componentwise)

$\rho(|\hat{A}|) u(|\hat{A}|)=|\hat{A}| u(|\hat{A}|) \geq|A| u(|\hat{A}|), \quad \rho(|\hat{A}|) v(|\hat{A}|)=v(|\hat{A}|)|\hat{A}| \geq v(|\hat{A}|)|A|$

where the left, resp. right, eigenvector $u(|\hat{A}|) \geq 0$, resp. $v(|\hat{A}|) \geq 0$. Observe that on premultiplying the first relation of $(2)$ by $v(|A|)$, we immediately conclude that $\rho(|\hat{A}|) \geq$ $\rho(|A|)$ for every $|\hat{A}| \neq|A|$.

Recall that the one norm (denoted $\|\cdot\|_{1}$ ) and the maximum (or infinity) norm (denoted $\left.\|\cdot\|_{\infty}\right)$ of the matrix $A=\left[a_{i j}\right]$ is given by $\max _{j} \sum_{i=1}^{r}\left|a_{i j}\right|$ and by $\max _{i} \sum_{j=1}^{r}\left|a_{i j}\right|$ respectively. Similarly, for every $u \in \mathbb{R}_{+}^{r}$ (i.e. $u>0$, where $u=\left[u_{1}, \ldots, u_{r}\right]$ ) the $u$-weighted maximum norm of the matrix $A$ is given by $\|A\|_{u}=\max _{i=1, \ldots, r} \sum_{j=1}^{r}\left|a_{i j}\right| \frac{u_{j}}{u_{i}}$, and hence the $u$-weighted maximum norm of a (column) vector $d \in \mathbb{R}^{r},\|d\|_{u}=\max _{i=1, \ldots, r}\left|d_{i}\right|$. It can be easily shown that $\|\cdot\|_{u}$ is indeed a norm, in particular, $\|A\|_{u} \geq 0$ with $\|A\|_{u}=0$ iff $A=0 ;\|\lambda A\|_{u}=|\lambda|\|A\|_{u}$ for every $\lambda \in \mathbb{R}$; and if $A, B \in \mathbb{R}^{r \times r}$, then $\|A+B\|_{u} \leq\|A\|_{u}+\|B\|_{u}$. Moreover, supposing that $A=\left[a_{i j}\right] \in A_{I}$ is irreducible, let $u>0$ be selected such that $\rho(|A|) u=|A| u$. Then $\|A\|_{u}=\rho(|A|)$ (observe that $\left.\sum_{j=1}^{r}\left|a_{i j}\right| \frac{u_{j}}{u_{i}}=\rho(|A|) \quad \forall i=1, \ldots, r\right)$. In case of reducible $A$, for an arbitrary small $\varepsilon>0$ we can find $u^{\varepsilon}>0$ such that $\rho(|A|)+\varepsilon \geq\|A\|_{u^{e}} \geq \rho(|A|)$.

Observe that if $A \in A_{I}$ is stable, it may happen that $\|A\|>1$ both for the one and the maximum norm. Consider e.g. $A_{I}=\left[\begin{array}{ll}0.8 & 9.2 \\ 0.001 & 0.8\end{array}\right]$, then $\|A\|_{1}=\|A\|_{\infty}=10$; however, calculating the eigenvalues we can conclude that the Perron eigenvalue is equal to $\rho(A)=0.8959$ and the corresponding right Perron eigenvector can be set to $u=\left[\begin{array}{ll}85.92 & 1\end{array}\right]$. Hence the weighted $u$-maximum norm of the matrix $\|A\|_{u}=0.8959$.

Stability criteria for time-invariant discrete systems based on examination of extreme matrices are summarized in the following proposition (unless otherwise stated, $\|\cdot\|$ denotes an arbitrary matrix norm):

Proposition 1 (cf. Mori and Kokame (1987), Juang et al. (1989a)).

The time-invariant discrete system (1) is globally asymptotically stable if there exists a matrix norm such that the norm of every extreme matrix in $A_{I}$ is less than one.

Now we present a stability criterion similar to that in Proposition 1 that is based on the dominating matrix $\hat{A}$ instead of matrix norms of extreme matrices. Recalling that $|\hat{A}| \geq|A|$ for every $A \in A_{I}$ the following Proposition 2 can be easily verified by iterating (2).

Proposition 2 The time-invariant discrete system (1) is globally asymptotically stable if 
the modulus matrix of the dominating matrix $\hat{A}$ is asymptotically stable, i.e. if $\rho(|\hat{A}|)<1$. Then $h=1-\rho(|\hat{A}|)$ is the stability margin of (1) and there exists a matrix norm such that the norm of every matrix $A \in A_{I}$ (and hence also every extreme matrix in $A_{I}$ ) is less than unity, i.e. the stability conditions due to Proposition 1 are fulfilled.

The following proposition slightly extends some of the results reported in Han and Lee (1994), in particular, the part concerning the stability margins is believed to be new. On the contrary to the original proof, observe that Proposition 3 also immediately follows by iterating (2) where time-varying matrix $A(k)$ replaces the matrix $A$, Corollary 1 and Corollary 2 follow then trivially.

Proposition 3 (cf. Han and Lee (1994) Theorem 1 and Lemma 1).

The time-varying discrete system (1) is globally asymptotically stable if $\rho(|\hat{A}|)<1$. Then $h=1-\rho(|\hat{A}|)$ is the stability margin of (1).

Corollary 1 (cf. Han and Lee (1994) Corollary 1 and Corollary 2).

(i) Let $C \geq|B|$. Then (1) is asymptotically stable if and only if $\rho(C)<1$.

(ii) Let $|B| \geq|C|$. Then (1) is asymptotically stable if $\rho(|B|)<1$.

From Corollary 1 we immediately obtain necessary and sufficient conditions for stability of nonnegative time-invariant discrete interval systems studied in Shafai et al. (1991) and in Chen (1993).

Corollary 2 (cf. Shafai et al. (1991) and Chen (1993) Theorem 1).

Let $B \geq 0$. Then (1) is globally asymptotically stable if and only if $\rho(C)<1$. In particular, also the time-invariant system given by (1) is globally asymptotically stable.

Of course, the obtained results are also closely connectcd with the necessary and sufficient conditions for the stability of time-varying interval matrices reported in Bauer et al. (1993). These results can be summarized as follows.

Proposition 4 (cf. Bauer et al. (1993) Theorem and Lemma).

The time-varying discrete system (1) is globally asymptotically stable if and only if there exists a finite $n_{0}$ such that $\left\|\prod_{k=0}^{n_{0}} A(k)\right\|<1 \quad \forall A(k) \in A_{I}, k=0,1, \ldots, n_{0}$, where $\|\cdot\|$ is reserved for one norm or for maximum norm. The above condition is fulfilled if and only if $\left\|\prod_{k=0}^{n_{0}} A(k)\right\|<1 \quad \forall A(k) \in \bar{A}_{I}, k=0,1, \ldots, n_{0}$.

In what follows, we extend the results of Propositions 1, 2,3,4 and present some useful connections between similarity transformations, matrix norms and spectral radii. The obtained results will enable to test stability using spectral radii of the modulus matrices arising by multiplying extreme matrices of the considered interval system. In particular, we suggest an algorithmic procedure for evaluating products of extreme matrices to obtain sufficient stability conditions for the time-varying discrete interval system (1) along with the corresponding stability margins. The obtained results and the algorithmic procedure are tested on five numerical examples taken from the literature. 


\section{MAIN RESULTS}

For the sake of brevity sometimes we shall set $P(n) \equiv \prod_{k=0}^{n-1} A(k)$ where $A(k) \in A_{I}$. Observe that $P(1) \in A_{I}$, denote by $\hat{P}(1) \equiv \hat{A}$ the dominating matrix and recall that $\rho(|P(\cdot)|)$, resp. $u(|P(\cdot)|)$, is reserved for the spectral radius of $|P(\cdot)|$, resp. its right Perron eigenvector.

Lemma 1 Let $n_{0}=1,2, \ldots$ and $u \in \mathbb{R}_{+}^{r}$ be fixed. Then there exists a matrix sequence $\left\{\hat{A}(k): \hat{A}(k) \in \vec{A}_{I}, k=0,1, \ldots, n_{0}-1\right\}$ (called $\left(n_{0}, u\right)$-dominating matrix sequence) such that $\left\|\hat{P}\left(n_{0}, u\right)\right\|_{u} \equiv\left\|\prod_{k=0}^{n_{0}-1} \hat{A}(k)\right\|_{u} \geq\left\|P\left(n_{0}\right)\right\|_{u} \equiv\left\|\prod_{k=0}^{n_{0}-1} A(k)\right\|_{u}$ for every matrix sequence $\left\{A(k): A(k) \in A_{I}, k \stackrel{k=0}{=0}, \ldots, n_{0}-1\right\}$.

Lemma 2 Let for some $n_{0}=1,2, \ldots$ there exists a matrix sequence $\{\hat{A}(k): \hat{A}(k) \in$ $\left.\bar{A}_{I}, k=0,1, \ldots, n_{0}-1\right\}$ (called $n_{0}$-dominating matrix sequence) such that $u\left(\left|\hat{P}\left(n_{0}\right)\right|\right)>0$ and $\rho\left(\left|\hat{P}\left(n_{0}\right)\right|\right) u\left(\left|\hat{P}\left(n_{0}\right)\right|\right) \geq\left|P\left(n_{0}\right)\right| u\left(\left|\hat{P}\left(n_{0}\right)\right|\right)$ for any $P\left(n_{0}\right) \equiv \prod_{k=0}^{n_{0}-1} A(k), A(k) \in \vec{A}_{I}$. Then $\rho\left(\left|\hat{P}\left(n_{0}\right)\right|\right) \geq \rho\left(\left|P\left(n_{0}\right)\right|\right)$ for an arbitrary matrix sequence $\left\{A(k): A(k) \in A_{I}, k=\right.$ $\left.0, \ldots, n_{0}-1\right\}$. Moreover, $\rho\left(\left|\hat{P}\left(n_{0}\right)\right|\right)=\left\|\hat{P}\left(n_{0}\right)\right\|_{\hat{u}}$ for $\hat{u}=u\left(\left|\hat{P}\left(n_{0}\right)\right|\right)$.

Theorem 1 The time-varying discrete system (1) is asymptotically stable if there exists a finite $n_{0}=1,2, \ldots$ such that $\rho\left(\left|\hat{P}\left(n_{0}\right)\right|\right)<1$ where $\hat{P}\left(n_{0}\right) \equiv \prod_{k=0}^{n_{0}-1} \hat{A}(k), \hat{A}(k) \in \bar{A}_{I}$ is an $n_{0}$-dominating matrix sequence. Then $h=1-\left(\rho\left(\left|\hat{P}\left(n_{0}\right)\right|\right)\right)^{\frac{1}{n_{0}}}$ is the stability margin of (1).

Remark 1 Observe that the "if" part of Theorem 1 for $n_{0}=1$ is identical with Proposition 3 and if in Lemma 1 the $u$-weighted maximum norm is replaced by one or maximum norm, Lemma 1 is identical with the Lemma in Bauer et al. (1993).

As a simple example shows, it need not hold that $(\rho(|\hat{P}(n+1)|))^{\frac{1}{n+1}} \leq(\rho(|\hat{P}(n)|))^{\frac{1}{n}}$ however, some monotonicity properties of suitable subsequences of $\left\{(\rho(|\hat{P}(n)|))^{\frac{1}{n}}\right\}$ are presented in Proposition 5.

Example. Let $A_{I}=A=\left[\begin{array}{rr}-0.5 & 0.5 \\ 0.5 & 0.5\end{array}\right]$. Obviously $\rho(A)=\sqrt{0.5}=0.7071$.

Then $|\hat{A}|=\left[\begin{array}{ll}0.5 & 0.5 \\ 0.5 & 0.5\end{array}\right]$ with $\rho(|\hat{A}|)=1, \hat{P}(2)=|\hat{P}(2)|=\left[\begin{array}{rr}0.5 & 0 \\ 0 & 0.5\end{array}\right]$ where $\rho(|\hat{P}(2)|)=0.5$, and $(\rho(|\hat{P}(2)|))^{\frac{1}{2}}=\sqrt{0.5}=0.7071$, however,

$\hat{P}(3)=\left[\begin{array}{rr}-0.25 & 0.25 \\ 0.25 & 0.25\end{array}\right] \Rightarrow|\hat{P}(3)|=\left[\begin{array}{ll}0.25 & 0.25 \\ 0.25 & 0.25\end{array}\right]$

and hence $\rho(|\hat{P}(3)|)=0.5 \Longrightarrow(\rho(|\hat{P}(3)|))^{\frac{1}{3}}=0.79$. 
Proposition 5 For every integer $k, n>1 \rho(|\hat{P}(1)|) \geq(\rho(|\hat{P}(n)|))^{\frac{1}{n}}$, and $\rho(|\hat{P}(n)|) \geq$ $(\rho(|\hat{P}(n \cdot k)|))^{\frac{1}{k}}$.

Now we present useful connections between matrix norms and spectral radii of matrix sequences. The symbol $\|\cdot\|$ is reserved for one norm or for maximum norm.

Theorem 2 For every $\varepsilon>0, n=1,2, \ldots$ and $P\left(n_{0}\right) \equiv \prod_{k=0}^{n_{0}-1} A(k)$, where $A(k) \in A_{I}$, there exists a nonsingular diagonal matrix $T^{\varepsilon}(n)$ such that $\left\|\left(T^{\varepsilon}(n)\right)^{-1} P(n) T^{\varepsilon}(n)\right\|-\varepsilon \leq$ $\rho(|P(n)|) \leq\left\|\left(T^{\varepsilon}(n)\right)^{-1} P(n) T^{\varepsilon}(n)\right\|$. Furthermore, if there exists a strictly positive Perron eigenvector of $|P(n)|$, say $u=\left[u_{i}\right]$ then $\left\|(T(n))^{-1} P(n) T(n)\right\|=\rho(|P(n)|)$ for $T(n)=$ diag $\left\{u_{i}\right\}$. In particular, $\rho(|P(n)|)<1$ if and only if there exists a nonsingular, diagonal matrix $T(n)$ such that $\left\|(T(n))^{-1} P(n) T(n)\right\|<1$.

Now we are in a position to present an algorithmical procedure for checking stability of time-varying interval systems.

\section{Algorithm 1}

Step 0 . Set $n=1$ and generate the set $\mathcal{P}(1) \equiv \bar{A}_{I}$ of all extreme matrices.

Step 1 . Find in the set $\mathcal{P}(1) \equiv \bar{A}_{I}$ the dominating matrix $\hat{P}(1) \equiv \hat{A}$ and calculate the spectral radius $\rho(|\hat{P}(1)|\}$ of the modulus of $\hat{P}(1)$. If $\rho(|\hat{P}(1)|)<1$ terminate with conclusion of stability and calculate the stability margin $h(1)=1-\rho(|\hat{P}(1)|)$ (if $\hat{P}(1)=$ $|\hat{P}(1)|$ this stability margin is the tightest possible one). In case that $\rho(|\hat{P}(1)|) \geq 1$ then check if also $|\hat{P}(1)| \in \bar{A}_{I}$ (i.e. $|\hat{P}(1)|=\hat{P}(1)$ ). If it is the case then terminate with conclusion of instability, else proceed to Step 2.

Step 2. Set $n=n+1$ and generate the set $\mathcal{P}(n)$ of all products of $n$ extreme matrices. Proceed to Step 3.

Step 3. Calculate the spectral radius $\rho(|P(n)|)$ of every matrix $|P(n)|$ where $P(n) \in$ $\mathcal{P}(n)$. Let $\hat{P}(n)=\prod_{k=0}^{n-1} \hat{A}(k) \in \mathcal{P}(n)$ be such that $u(|\hat{P}(n)|)>0$ and $\rho(|\hat{P}(n)|) u(|\hat{P}(n)|) \geq$ $|P(n)| u(|\hat{P}(n)|)$ for every $P(n) \in \mathcal{P}(n)$. If $\rho(|\hat{P}(n)|)<1$ then terminate with conclusion of stability and calculate the stability margin $h(n)=1-\left(\rho(|\hat{P}(n)|)^{\frac{1}{n}}\right.$ (if $\hat{P}(n)=|\hat{P}(n)|$ this stability margin is the tightest possible one), else proceed to Step 2.

\section{ILLUSTRATIVE EXAMPLES}

Finally, we shall test Algorithm 1 on five numerical examples of linear interval systems borrowed from Bauer et al. (1993), Han and Lee (1994), Kolla et al. (1989) and of Juang and Shao (1989).

Example 1 (see Example 1 in Bauer et al. (1993)).

Consider a discrete-time dynamic system with the following interval matrix

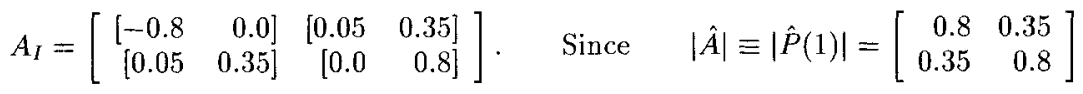


is not stable and $|\hat{A}| \notin \bar{A}_{I}$, we calculate $P(2)$ 's (products of two extreme matrices) and find that $|\hat{P}(2)|=\left[\begin{array}{rr}0.0 & 0.35 \\ 0.35 & 0.8\end{array}\right]^{2}=\left[\begin{array}{rr}0.1225 & 0.28 \\ 0.28 & 0.7625\end{array}\right], \quad u(|\hat{P}(2)|)=\left[\begin{array}{l}0.375 \\ 1.000\end{array}\right]$, where $\hat{P}(2)$ is a dominating matrix sequence. Even if $\|\hat{P}(2)\|_{1}=\|\hat{P}(2)\|_{\infty}=1.043$, for the spectral radius of $|\hat{P}(2)|$ we get $\rho(|\hat{P}(2)|)=0.8676$. Hence the system is stable and the resulting stability margin $h=1-(0.8676)^{\frac{1}{2}}=0.0685$.

Example 2 (see Example 2 in Bauer et al. (1993)).

Consider a discrete-time dynamic system with the following interval matrix

$A_{I}=\left[\begin{array}{rrr}0.7 & {[-0.2} & 0.45 \\ 0.45 & {[-0.1} & 0.2\end{array}\right] \quad$ Obviously, $\quad \hat{A} \equiv \hat{P}(1)=\left[\begin{array}{rr}0.7 & 0.45 \\ 0.45 & 0.2\end{array}\right]$

with $\|\hat{P}(1)\|_{\infty}=\|\hat{P}(1)\|_{1}=1.15$ and moreover $|\hat{P}(1)|=\hat{P}(1) \in \bar{A}_{I}$ with $\rho(|\hat{P}(1)|)=$ 0.967 . Hence the considered system is stable with stability margin 0.032 that is the tightest possible one.

Example 3 (see Example 3 in Juang and Shao (1989)).

Consider a discrete-time dynamic system with the following interval matrix

$A_{I}=\left[\begin{array}{lll}{[-0.20} & 0.16\end{array}\right]\left[\begin{array}{ll}-0.34 & 0.02\end{array}\right] . \quad$ Then $\quad|\hat{A}| \equiv|\hat{P}(1)|=\left[\begin{array}{rr}0.2 & 0.34 \\ 0.24 & 0.2\end{array}\right]$

$\|\hat{P}(1)\|_{1}=\|\hat{P}(1)\|_{\infty}=0.54$ and $\rho(|\hat{P}(1)|)=0.485$. Hence the considered system is stable with stability margin at least 0.515 (since $|\hat{P}(1)| \notin \bar{A}_{I}$ this stability need not be the tightest possible one).

Example 4 (see Example 1 in Han and Lee (1994)).

Consider a discrete-time dynamic system with the following interval matrix

$A_{I}=\left[\begin{array}{rrr}{[-0.7} & 0.6] & 0.6 \\ 0.1 & & 0.5\end{array}\right] . \quad$ Then $\quad|\hat{A}| \equiv|\hat{P}(1)|=\left[\begin{array}{ll}0.7 & 0.6 \\ 0.1 & 0.5\end{array}\right]$

$\|\hat{P}(1)\|_{1}=1.1,\|\hat{P}(1)\|_{\infty}=1.3$ however $\rho(|\hat{P}(1)|)=0.8646,|\hat{P}(1)| \notin \bar{A}_{I}$. Hence the considered system is stable with stability margin at least 0.1354 .

Example 5 (see Example 3 in Kolla et al. (1989)).

Consider a discrete-time dynamic system with the following interval matrix

$A_{I}=\left[\begin{array}{rrr}{[-0.5} & 0.5\end{array}\right]\left[\begin{array}{ll}0 & 0.6\end{array}\right] . \quad$ Then $\quad \hat{A} \equiv|\hat{P}(1)|=\hat{P}(1)=\left[\begin{array}{rr}0.5 & 0.6 \\ 0.75 & 0\end{array}\right]$

$\|\hat{P}(1)\|_{1}=1.25,\|\hat{P}(1)\|_{\infty}=1.1$, but $\rho(\mid \hat{P}(1) \|)=0.966$. Hence the considered system is stable with stability margin 0.034 that is the tightest possible one. 


\section{REFERENCES}

Bauer, P.H., Premaratne, K., and Durán, J. (1993) A necessary and sufficient condition for robust asymptotic stability of time-variant discrete systems. IEEE Trans. Automat. Control, AC-38, 1427-30.

Berman, A. and Plemmons, R.J. (1979) Nonnegative Matrices in the Mathematical Sciences. Academic Press, New York.

Chen, J. (1993) Comments on 'A necessary and sufficient conditions for the stability of nonnegative interval discrete systems.' IEEE Trans. Automat. Control, AC-38, 189.

Gantmakher, F.R. (1966) Teoriya matric. Second edition. Nauka, Moscow. English translation: The Theory of Matrices, Volume I, II. Chelsea, New York 1959.

Han, H.S. and Lee, J.G. (1994) Necessary and sufficient conditions for stability of timevarying discrete interval matrices. Internat. J. Control, 59, 1021-9.

Internat. J. Control (1989) 49, 1095-1108, Correspondence.

Jiang, C.L. (1988) Sufficient and necessary condition for the asymptotic stability of discrete linear interval systems. Internat. J. Control, 47, 1563-5.

Juang, Y.-T. and Shao, C.-S. (1989) Stability analysis of dynamic interval systems. Internat. J. Control, 49, 1401-8.

Juang, Y.-T., Tung, S.-H., and Ho, T.-C. (1989a) Sufficient condition for asymptotic stability of discrete interval systems. Internat. J. Control, 49, 1799-803.

Kolla, S.R. and Farison, J.B. (1988) Counterexamples to 'Sufficient and necessary condition for the asymptotic stability of discrete linear interval systems.' Internat. J. Control, 48, 1751-2.

Kolla, S.,R., Yedavalli, R.K., and Farison, J.B. (1989) Robust stability bounds on timevarying perturbations for state-space models of linear discrete-time systems. Internat. J. Control, 50, 151-9.

Mori, T. and Kokame, H. (1987) Convergence properties of interval matrices and interval polynomials. Internat. J. Control, 45, 243-8.

Shafai, B., Perev, K., Cowley, J., and Chehab, Y. (1991) A necessary and sufficient conditions for the stability of nonnegative interval discrete systems. IEEE Trans. Automat. Control, AC-36, 742-6. 